\title{
Políticas MUNICIPALES de GÉNERO EN CATALUNYA Y LA LOMBARdía. \\ ESTUDIO DEL CAMBIO INSTITUCIONAL Y ORGANIZATIVO \\ REALIZADO PARA FACILITAR SU IMPLEMENTACIÓN ${ }^{1}$.
}

\author{
Mònica Gelambí Torrell
}

\section{Resumen}

El artículo analiza las coincidencias y divergencias existentes en los cambios institucionales que los ayuntamientos catalanes y lombardos han realizado para facilitar la introducción de la perspectiva de género. Para estudiar estos cambios se han analizado tres ámbitos: el proceso político, los mecanismos políticos y los actores que intervienen en el diseño de las políticas. Los resultados se han obtenido del estudio en profundidad de seis ayuntamientos de cada territorio que ya están realizando políticas de género. Las conclusiones obtenidas constatan la presencia de cambios y demuestran cómo las estrategias seguidas son distintas en ambos casos, aunque se comparta la filosofía existente detrás de las políticas de género.

Palabras clave: políticas de género, políticas municipales, Catalunya, Lombardía

\begin{abstract}
This article analyses the institutional changes to assess the progress of gender mainstreaming in Catalonia and Lombardia. The research centred on case studies of how gender in being integrated into policy in the following three aspects: the policy process, political tools and actors involved in public policy. The comparative analysis of gender equality policies in six Catalan and Italian cities indicated that the development of gender mainstreaming makes changes in the local governments. These changes are different in the two regions but they used the same concept of gender mainstreaming.
\end{abstract}

Key words: gender equality policies, local public policies, Catalonia, Lombardia

\footnotetext{
${ }^{1}$ Este artículo es el resultado de la investigación realizada gracias a la Beca para la formación de Estudios de Postgrado en Universidades y Centros Superiores de Investigación de la Unión Europea de la Fundación Caja Madrid.
} 


\section{Introducción}

Los Estados miembros de la Unión Europea se han comprometido, como indica el Plan de Trabajo para la igualdad entre mujeres y hombres 2006-2010 de la Comisión, a trabajar para lograr la igualdad de oportunidades y hacer desaparecer toda discriminación a través de un enfoque dual de la igualdad en género ${ }^{2}$. Esta perspectiva dual promueve la introducción de la gestión transversal en todas las políticas de todos los niveles, al mismo tiempo que se continúan realizando medidas específicas dirigidas a las desigualdades de género (políticas 'tradicionales' de igualdad de género).

A pesar de existir un claro compromiso por parte de los actores políticos a favor de esta estrategia global, introducir la gestión transversal de género en el proceso de elaboración de las políticas no es fácil. La definición que ha elaborado el Consejo Económico y Social de la Unión Europea evidencia la dificultad que comporta esta tarea:

La perspectiva de la gestión transversal de género es un proceso que implica tener en cuenta las implicaciones que cualquier actuación planificada tiene para las mujeres y para los hombres, incluyendo legislación, políticas o programas, en todas las áreas y en todos los niveles. Es una estrategia para que todas las experiencias e intereses de las mujeres y los hombres sean una dimensión integrada en el diseño, implementación, monitorización y evaluación de políticas y programas en todas las esferas sociales, económicas y políticas, de manera que la cualidad en los beneficios y en las desigualdades entre las mujeres y los hombres no sean perpetuadas. El objetivo final es lograr la equidad de género ${ }^{3}$.

\footnotetext{
2 "El género es una definición de las mujeres y los hombres construida socialmente. Es el diseño social de un sexo biológico, determinado por la concepción de tareas, funciones y roles atribuidos a las mujeres y a los hombres en la sociedad y en la vida pública y privada. Se trata de una definición específica cultural de la femineidad y de la masculinidad y varía por tanto en el tiempo y en el espacio. La construcción y la reproducción del género tiene lugar tanto a nivel individual como a nivel social". Conclusiones de la Conferencia sobre "Igualdad y democracia: Utopía o desafío", Consejo de Europa 1995. Grupo de Expertos 1999: 12.

${ }^{3}$ Conclusiones convenidas por el ECOSOC 1997/2. Naciones Unidas. Comité Económico y Social, 1997. (DOC \#A text from ECOSOC 23).
}

Cuestiones de género, 2008, nº 3, pp. 367/402 
Políticas municipales de género en Catalunya y la Lombardía...

Con el objetivo de ayudar en este proceso, el Grupo de expertos del Consejo de Europa ha resaltado cinco elementos clave para poder implementar la estrategia de la gestión transversal de género:

1) la ampliación del concepto de igualdad de género, que tiene que ir más allá de las políticas de acción positiva;

2) la incorporación de la perspectiva de género en la agenda política dominante;

3) la inclusión y la participación de las mujeres en las instituciones y en los procesos de toma de decisiones;

4) la valoración como importantes y prioritarias de las políticas de igualdad de género y de aquellas que tienen especial relevancia para las mujeres;

5) el cambio en las culturas institucional y organizativa de las organizaciones responsables (Lombardo, 2003: 7-8).

El estudio realizado, cuyas conclusiones se presentan en este artículo, se centra en el quinto elemento clave para la implementación de la gestión transversal de género: el cambio institucional y organizativo de los ayuntamientos catalanes y lombardos.

El cambio institucional y organizativo necesario para implementar la estrategia del 'gender mainstreaming' afecta, según el mismo Grupo de especialistas (1999: 32), los siguientes tres ámbitos:

- el proceso político, que se verá obligado a introducir la dimensión de género en la elaboración de toda política;

- los mecanismos políticos, que tendrán que incorporar instrumentos y técnicas que faciliten un trabajo transversal con dimensión de género;

- $\quad$ los actores políticos, que cambiaran por la apertura del debate a una sociedad civil más amplia y con una mayor representación femenina.

http://www.un.org/documents/ecosoc/docs/1997/e1997-66.htm 


\section{Selección del objeto de estudio y métodos de investigación}

El estudio realizado se centra en las políticas de igualdad en género de los ayuntamientos de más de 20.000 habitantes de la comunidad autónoma de Catalunya en España y de la región de la Lombardía en Italia. Tanto España como Italia se presentan como ejemplos del régimen de bienestar mediterráneo o del Sur de Europa (Subirats i Gomà, 2000: 39-40; Trifiletti, 1999; Ferrera, 2005). En ambos casos se otorga un rol esencial a la familia como prestadora de servicios, y se reserva al Estado un papel básicamente residual sobre las responsabilidades de cuidado. El resultado que se obtiene es un modelo de solidariedad familiar y parental con importantes consecuencias para la vida de las mujeres ${ }^{4}$ (Naldini, 2000: 103; de León, 2002: 160). Ambas regiones comparten, consecuentemente, el mismo orden de género 5 .

El trabajo empírico en que se basa este artículo consistió en entrevistas en profundidad semi-estructuradas realizadas a las personas responsables de las políticas para la igualdad de oportunidades y de género. Esta responsabilidad recae en la figura de los concejales en Catalunya y de los 'asesores' en la Lombardía, en ambos casos se trata de una persona que recibe el encargo por parte del alcalde y que forma parte de la equipo de gobierno de la ciudad.

En un inicio me puse en contacto con los ayuntamientos de las ciudades con más de 20.000 habitantes, haciendo un total de 45 entrevistas en Catalunya y 19 en la Lombardía. Esta primera aproximación me permitió realizar un esbozo del concepto de políticas de igualdad de oportunidades y de género que estaban utilizando, pudiendo

\footnotetext{
${ }^{4}$ Naldini (2000: 99) afirma en su artículo que aún en los años noventa España e Italia comparten un modelo alargado de 'male breadwinner' porque incluye la dependencia no solamente de la mujer y los hijos, sino también de otros familiares 'a cargo'.

5 Por "orden de género" se entiende el sistema socialmente compartido de normas, principios, costumbres y políticas que establecen la distribución de derechos, tareas y oportunidades de vida para ambos sexos. La división de roles entre personas que se dedican al trabajo retribuido y las que cumplen el trabajo reproductivo gratuito es parte de un complejo sistema social de precedencia y subordinación. (Lombardo 2003: 67-8)

Cuestiones de género, 2008, nº 3, pp. 367/402
} 
Políticas municipales de género en Catalunya y la Lombardía...

diferenciar entre aquellos que realizaban políticas específicas de igualdad de género (parten de las desigualdades que caracterizan las relaciones reales entre hombres y mujeres y centran su atención en cómo se tiene que superar la desigualdad), de aquellos que estaban trabajando con la idea del gender mainstreaming, sin dejar de realizar, en la mayoría de los casos, medidas específicas dirigidas a las mujeres (Gelambí, 2005).

En un segundo momento seleccioné 6 ayuntamientos de cada región para un análisis más exhaustivo, escogiéndolos al azar entre los que habían introducido en su discurso político la idea de gestión transversal de género y ya estaban trabajando en esta línea ${ }^{6}$. Además de las entrevistas se han tenido en cuenta los documentos y las publicaciones realizadas por los ayuntamientos en este ámbito.

Los municipios escogidos no son representativos estadísticamente. Por lo tanto, los resultados de esta investigación son indicativos, sugieren algunas regularidades en los cambios experimentados por los ayuntamientos catalanes y lombardos en el proceso de implementación de la gestión transversal de género, pero carecen de valor estadístico.

Se trata de un estudio cualitativo que quiere entender cómo se está introduciendo la perspectiva de género en el ámbito municipal, sin querer realizar una generalización de los resultados obtenidos.

\section{El cambio institucional y organizativo necesario para realizar políticas de género en el ámbito local}

${ }^{6}$ Los municipios integrantes del estudio son los siguientes. De Catalunya: Girona, Manresa, Mataró, Sant Feliu de Llobregat, Vilafranca del Penedès y Vilanova i la Geltrú. De la Lombardia: Bergamo, Brescia, Gallarate, Monza, Pavia y Vigevano. Las entrevistas fueron realizadas en Catalunya entre el mes de noviembre 2002 y el mes de febrero 2003; y en la Lombardía entre el mes de enero 2005 y el mes de enero 2006. 


\section{1. Cambio en el proceso político}

Para analizar si se ha producido o no un cambio en el proceso político con el objetivo de introducir la perspectiva de género en las políticas municipales se han seguido las indicaciones dadas por las Naciones Unidas. Esta Organización, en base a su propia experiencia de más de 10 años, ha destacado tres aspectos como los más importantes cuando se quiere utilizar la estrategia de mainstreaming de género.

- La presencia de un enfoque activo, ya que no se trata de un proceso que se realice de forma automática o natural. Es imprescindible marcarse el objetivo de introducir en todas las intervenciones la atención de género, en caso contrario es muy difícil que realmente se produzca.

- La formulación de un compromiso político explícito con la implementación de la gestión transversal de género. Realidad inexistente cuando los temas de igualdad de género no son claramente apreciables o no influyen en los procesos críticos de toma de decisiones;

- La atención prestada a la igualdad de género tiene que ser sistemática y permanente. La transversalidad de género no debería limitarse a los sectores sociales donde las contribuciones y las necesidades de las mujeres estén bien establecidas, o a los componentes más "flexibles" de los programas y los proyectos. El mainstreaming debería aplicarse en todos los tipos de intervenciones y en todos los niveles.

En resumen, la introducción de la gestión transversal de género tiene que ser el resultado de un enfoque ACTIVO, EXPLÍCITO y SISTEMÁTICO. Y con el objetivo de comprobar si es así en los ayuntamientos catalanes y lombardos se han analizado los siguientes aspectos:

- Activo: si se trata de un proyecto municipal (de todo el ayuntamiento) o de una iniciativa del político/a responsable del ámbito de las políticas de igualdad de oportunidades o de la mujer.

- Explícito: si se ha consolidado el compromiso municipal en algún documento público o no.

- Sistemático: si la estrategia de mainstreaming de género se limita a los sectores sociales o se ha ampliado a todas las intervenciones municipales. 
Políticas municipales de género en Catalunya y la Lombardía...

\section{III.2. Cambio en los mecanismos políticos}

El estudio ha analizado qué herramientas y técnicas están utilizando los ayuntamientos catalanes y lombardos para facilitar la introducción de la gestión transversal de género. Se parte de la consideración que tanto las Concejalías de la Mujer como los Planes de igualdad se presentan como instrumentos tradicionales en el ámbito de las políticas para la igualdad de oportunidades entre hombres y mujeres (Instituto de la Mujer 2005; Valiente 1999 y 2006; Bustelo, 2004).

Los organismos de igualdad han asumido un rol activo en la evolución de las políticas de igualdad de oportunidades entre mujeres y hombres, favoreciendo la introducción de referencias de género en las discusiones políticas, como demuestra Celia Valiente (1999a: 173):

Desde la Segunda Guerra Mundial, pero especialmente desde los años 70, se han fundado en todos los países occidentales, y en muchos no occidentales, instituciones que tienen como principal objetivo impulsar medidas que mejoren el estatus de las mujeres, y que contribuyan a equiparar su situación a la de los hombres. Este conjunto de organismos de igualdad, existente en todos los ámbitos territoriales del Estado, se denomina en la literatura de las ciencias sociales "feminismo de Estado" (state feminism), "Feminismo oficial" o "feminismo institucional".

La utilidad de los Planes de Igualdad de Oportunidades entre mujeres y hombres la podemos encontrar en la misma Unión Europea, que inicia a diseñar sus planes de acción en el momento que decide transformar las medidas aisladas que realizaba a favor de las mujeres en un enfoque más integrador. Los planes son instrumentos políticos de actuación, que después de analizar la realidad sobre la que se quiere intervenir mediante la elaboración de un diagnóstico de género, deciden las mejores acciones o estrategias que se pueden utilizar para lograr los objetivos políticamente decididos. Concretan al máximo las "acciones positivas" que se tienen que desarrollar para corregir las desigualdades entre hombres y mujeres, especificando su espacio de acción y el periodo temporal para su implementación (Arranz, 2000: 39). 
Para conocer si se han producido cambios en los mecanismos políticos municipales para facilitar la implementación de la gestión transversal de género se han analizado los siguientes aspectos:

- Si se han introducido cambios en el ORGANIGRAMA MUNICIPAL para facilitar la introducción de esta perspectiva. En relación a los ayuntamientos que han optado por crear organismos de igualdad se analizarán tres características que, según la literatura sobre feminismo de Estado, previsiblemente influyen en el impacto político de dichas instituciones: la prioridad que otorgan a una materia; su presupuesto y plantilla y si su dirección se encuentra próxima o distante al movimiento de mujeres (Valiente 2006: 22).

- Si se ha elaborado un PLAN DE ACTUACIÓN que estructure el proceso de implementación.

- La tipología de políticas que se están realizando para asegurar la igualdad de oportunidades en género en cada una de las ciudades estudiadas. Las políticas realizadas por las administraciones se dividirán en tres grandes grupos: políticas dirigidas exclusivamente a las mujeres; políticas para fomentar y facilitar la implementación transversal de género; y políticas de género, entendidas como aquellas políticas que se han diseñado considerando la situación en la que se encuentran los hombres y las mujeres de la ciudad ${ }^{7}$.

\section{III.2 Cambio en los actores}

La presencia de cambio en los actores deriva de la complejidad que lleva apareada la transversalidad en sí misma. Esta situación es el resultado de la profunda transformación histórica que han experimentado las ciudades como consecuencia de las nuevas tecnologías y del proceso de globalización de la economía y de la comunicación (Borja y Castells, 1997: 27). En este proceso de globalización la identidad local se está revalorizando (Borja y Castells, 1997: 3; Blanco y Gomà 2002: 18), y la participación

\footnotetext{
${ }^{7}$ Todo el estudio se basa en la convicción que todas las políticas de igualdad no son políticas de género. Como expone muy claramente Varella Martí (2001) en relación al ámbito del trabajo: "no cualquier política de igualdad tendrá impactos transformadores, en tanto que superadores de las desigualdades que afectan a las mujeres en el ámbito del empleo. Los impactos de las políticas de igualad también pueden ser reproductores o reforzadores de la desigual posición que las mujeres ocupan respecto a los hombres en el mercado de trabajo".
}

Cuestiones de género, 2008, nº 3, pp. 367/402 
Políticas municipales de género en Catalunya y la Lombardía...

ciudadana se está convirtiendo en un elemento clave del cambio. Como explica Borja en su libro Estado y ciudad: descentralización política y participación (1988) las ciudades no tendrían la fuerza necesaria para poder navegar en los circuitos globales sin la existencia de una relación sólida entre la ciudadanía y sus gobiernos.

Cambio que expresan los propios protagonistas del ámbito político municipal, como es posible observar en el estudio realizado por Brugué (2002: 27) sobre las transformaciones experimentadas por los concejales catalanes:

Los concejales de los noventa se replantean el equilibrio entre "la necesidad de escuchar a todos" y la "obligación de mantener la última palabra". Coinciden con los concejales de los ochenta en la necesidad de escuchar a los ciudadanos y a sus agrupaciones, aunque empiezan a añadir la posibilidad de incorporarlos al proceso decisional. De este modo, los estilos de gobierno asumirían la dimensión relacional al introducir lógicas de negociación, pacto y compromiso, tanto en el diseño como en la ejecución de las políticas locales.

Para conocer los cambios que se han producido en los actores que participan en el proceso de elaboración de las políticas de género el estudio ha analizado dos aspectos:

1.El rol que se atribuye a la participación ciudadana en la estrategia municipal del mainstreaming de género.

2. Si se han creado o no organismos de participación ciudadana que faciliten una relación permanente entre la ciudadanía y el gobierno de la ciudad.

\section{La introducción de la perspectiva de género en los ayuntamientos de Catalunya}

\section{y Lombardía}

\section{1 Presencia de cambios en el proceso político}

\section{a) Compromiso ACTIVO}

Todos los ayuntamientos catalanes estudiados manifiestan que la incorporación de la perspectiva de género forma parte de su proyecto municipal. Es posible atestiguar que existe un compromiso general de todo el equipo de gobierno a favor del 'gender 
mainstreaming'. Afirmación realizada con importantes precisiones, ya que la presencia del compromiso general no asegura su cumplimiento a nivel individual. En la mayoría de casos que se introduzca realmente la visión de género en el diseño de las políticas depende de la sensibilidad concreta de cada concejal o concejala, recayendo sobre la concejalía de la mujer o de políticas de igualdad (cuando existen) el rol de promotor activo del compromiso asumido.

La dualidad en el pacto municipal se observa claramente en las palabras de una de las concejalas entrevistadas, que comenta que, a pesar de existir el compromiso de todo el ayuntamiento, la que se encarga de realizar políticas de igualdad en género es la concejalía de la mujer:

Plan de actuación municipal para el año 2000 (...) Se preveían todas las acciones que se tenían que hacer en todas las concejalías. Se realizó un apartado dirigido a las políticas de mujeres. El proceso de decisión se realizó mediante debate público. Cada concejalía propuso objetivos y acciones y se discutieron en el proceso de participación (...) La política de la mujer la lleva la concejala, soy yo la que me encargo de realizar estas políticas. Es un trabajo muy personal. (...) Cada vez hay más sensibilidad y más conocimiento de la realidad. Está evolucionando, los concejales escuchan y se están logrando resultados políticamente. Existe una percepción positiva de la evolución de la perspectiva de género ${ }^{8}$.

Los ayuntamientos de la Lombardía estudiados no mantienen una postura uniforme en este tema. Solamente dos municipalidades afirman que se trata de un proyecto de todo el ayuntamiento. Los otros cuatro lo definen como un encargo del alcalde que recae exclusivamente en manos de la persona responsable del área de la mujer o de políticas de igualdad de oportunidades. En los dos ayuntamientos que se ha articulado el compromiso general se realizan las mismas apreciaciones que en el caso catalán, atribuyendo a una concejalía la responsabilidad final de promover y supervisar

\footnotetext{
${ }^{8}$ La traducción del italiano y del catalán de las entrevistas ha sido realizada por la propia autora del artículo. Ayuntamiento catalán de izquierdas de una ciudad mediana (con una población entre los 50.000 y los 100.000 habitantes).
}

Cuestiones de género, 2008, nº 3, pp. 367/402 
Políticas municipales de género en Catalunya y la Lombardía...

la realización de políticas de género. Una de las responsable lo expone con las siguientes palabras:

En las líneas programáticas que como Junta estamos predisponiendo hay un capítulo relativo concretamente a la ciudad de las mujeres, donde se dice sustancialmente que la visión de género es un elemento que distingue toda la política de la administración, no es una cuestión limitada a las mujeres, se quiere asumir esta visión de género al interior de todas las políticas de la administración, y esto me parece una cosa muy importante ${ }^{9}$.

\section{b) Compromiso EXPLÍ́CITO}

En este aspecto se aprecia una posición bien diferenciada entre los ayuntamientos catalanes y lombardos. La mayoría de los consistorios catalanes (4 de los 6) han externalizado su compromiso mediante la elaboración de un plan de igualdad de oportunidades entre mujeres y hombres, instrumento inexistente en el ámbito municipal lombardo. Solamente uno de los dos ayuntamientos estudiados de la Lombardía ha concretado en un documento el compromiso municipal favorable a la gestión transversal de género; y lo ha hecho mediante su incorporación en las líneas programáticas de la ciudad. Esta estrategia también ha sido utilizada por uno de los ayuntamientos catalanes que manifiesta haber introducido un apartado de políticas de mujeres dentro del Plan Estratégico de actuación municipal. Finalmente, tenemos un caso catalán y otro lombardo en el que no se ha realizado ningún compromiso explícito, argumentando por parte del ayuntamiento catalán que se ha logrado el compromiso informal de todas las áreas mediante el acuerdo con sus responsable políticos de participar en el proceso formativo existente en el ayuntamiento; y por parte del consistorio lombardo mediante la directriz política claramente dada por el alcalde a todos sus asesores, que se considera en sí misma vinculante.

\footnotetext{
${ }^{9}$ Ayuntamiento lombardo de izquierdas de una ciudad grande (con una población entre 100.000 y 150.000 habitantes).
} 


\section{c) Cambio sistemático}

Las concejalías catalanas que se encargan de la implementación de políticas de género manifiestan que se trata de un proceso lento y que aún no se ha producido en todas las áreas. Consideran como factor determinante la sensibilidad de género que tienen de las personas responsables de los distintos departamento, sin que tenga que ser relevante su ámbito concreto de actuación. En palabras de una concejala:

En esto las personas son muy importantes, el buen funcionamiento depende mucho de la voluntad individual y del hecho de creer en el proyecto. Los ámbitos aquí han jugado poco, no cuesta porque ha habido muchas concejalías, es decir, que no ha sido determinante que se trate de un ámbito considerado tradicionalmente como masculino, porque la historia del ayuntamiento no ha sido asi ${ }^{10}$.

A pesar de compartir esta convicción general, una de las personas entrevistadas remarca que existe una mayor sensibilidad por parte de las mujeres con responsabilidades políticas:

No todo el mundo se lo cree de la misma manera, los hombres menos que las mujeres. Es difícil porque el tema mujeres es como de segundo nivel ${ }^{11}$.

Es posible apreciar como en algunos casos las personas más sensibilizadas son las responsables de los ámbitos que tradicionalmente se han definido como 'femeninos' (ámbitos sociales):

El problema que se plantea es que las concejalías no están acostumbradas a trabajar la visión de género. Esto produce que sea difícil el trabajo transversal (...) Se ha trabajado sobretodo con la concejalía de educación y de cultura. ${ }^{12}$

\footnotetext{
${ }^{10}$ Ayuntamiento catalán de izquierdas de una ciudad pequeña (con una población entre los 20.000 y los 50.000 habitantes).

${ }^{11}$ Ayuntamiento catalán de izquierdas de una ciudad pequeña.

${ }^{12}$ Ayuntamiento catalán de izquierdas de una ciudad mediana.

Cuestiones de género, 2008, nº 3, pp. 367/402
} 
Políticas municipales de género en Catalunya y la Lombardía...

Los ayuntamientos lombardos presentan una mayor diversidad en las respuestas. Por un lado, los dos casos que han manifestado la existencia de un compromiso político de todo el ayuntamiento insisten en que se está trabajando desde todas las áreas, pudiendo afirmar que, a pesar de las dificultades existentes se trata de un proyecto sistemático. En palabras de una de las responsables:

De parte de algunos asesores ninguna, todo lo contrario, una fuerte implicación porque tengo que decir que somos un equipo, uno de los valores adjuntos de esta administración es haber creado por parte del acalde, de los partidos que han ganado estas elecciones administrativas una fuerte atención a trabajar en equipo, a trabajar transversalmente, de manera que hay una buena participación. ${ }^{13}$

Por otro lado, los ayuntamientos en los que las políticas de género recaen en manos de una responsable (persona que ha recibido el encargo del alcalde) muestran dos tendencias. Aquellos que remarcan que se trabaja con los ámbitos llevados por personas sensibilizadas, que en muchos casos son las responsables de los ámbitos que tradicionalmente se han considerado más cercanos a los problemas de las mujeres:

Es más fácil relacionarse con aquellos asesores responsables de áreas como educación o servicios sociales porque sin ninguna duda las mujeres conocen más estas problemáticas, lo que hace que sea más fácil. ${ }^{14}$

$\mathrm{Y}$ aquellos en los que se niega la existencia de colaboración de los otros asesores. Esta posición es claramente observable en la respuesta dada por una asesora cuando se le pregunta cuál es la reacción de los otros asesores cuando les realiza propuestas: "A los otros asesores no les importa nada, absolutamente" ${ }^{15}$

\footnotetext{
${ }^{13}$ Ayuntamiento lombardo izquierdas de una ciudad grande.

${ }^{14}$ Ayuntamiento catalán de izquierdas de una ciudad mediana.

${ }^{15}$ Ayuntamiento lombardo de derechas de una ciudad mediana.
} 
Una de las personas entrevistadas justifica el poco interés de los asesores/as con las siguientes palabras: "Aún existe la idea que las políticas al femenino y de igualdad de oportunidades son cosas de feministas."

\section{2 Cambios producidos en los mecanismos políticos}

\section{a) Organigrama municipal}

A nivel organizativo existen importantes diferencias entre los ayuntamientos catalanes y lombardos estudiados. En el caso catalán la mayoría (4 de 6 ) han optado por crear un órgano transversal para facilitar la implementación de la perspectiva de género, mientras que en el caso Lombardo la opción más recurrente (4 de 6) es la creación de un concejalía específica responsable de este ámbito político (Assessorato per le pari opportunità).

Cada uno de los ayuntamientos catalanes que ha elaborado un instrumento transversal ha realizado una opción diferente:

$>$ Creación de una concejalía transversal que se ocupa de 'juventud y mujeres'. Se caracteriza por no tener capacidad de gestión directa, su labor se concentra en decidir y promover los programas transversales que serán implementados por las concejalías 'tradicionales'. Así nos lo cuenta su responsable política:

Se han realizado diferentes programas transversales: jóvenes, niños, adolescentes, mujeres, inmigrantes, etc. Estos programas han sido diseñados por las concejalías transversales, que deciden los programas, pero no tienen capacidad de gestión y ésta es realizada de forma coordinada entre las otras concejalías y servicios. Es necesario que los diferentes departamentos se coordinen. (...) A la práctica tener una concejalía que se dedica solamente al diseño puede suponer problemas en la configuración de la propia concejalía ${ }^{17}$.

\footnotetext{
${ }^{16}$ Ayuntamiento lombardo de derecha de una ciudad pequeña.

${ }^{17}$ Ayuntamiento catalán de izquierdas de una ciudad grande.

Cuestiones de género, 2008, nº 3, pp. 367/402
} 
Políticas municipales de género en Catalunya y la Lombardía...

Constitución de la figura de Técnico de Planes Transversales. El encargo se ha dado a una concejalía que ya existía (concejalía de servicios sociales) porque se considera que la creación de una concejalía específica podía ser contraproducente con la idea de transversalidad. El técnico tiene que coordinar el personal administrativo de las otras áreas para realizar trabajos conjuntos e incentivar la introducción de la perspectiva de género en todos los departamentos. El ayuntamiento lo resume de la siguiente manera:

No existía ninguna planificación de las áreas, ni programas y se apostó por un técnico transversal, un político responsable y el compromiso de todas las áreas del ayuntamiento para llevar a cabo esta transversalidad. ${ }^{18}$

Diseño de una Oficina de Planes de Acción Ciudadana dentro del Área de Servicios Personales. En palabras de la concejala responsable de la Oficina:

Hay unas directrices políticas decididas por el equipo de gobierno. Estas directrices se pasaron a un técnico coordinador, para que se pusiera en contacto con los técnicos de las distintas áreas y planificaran conjuntamente las actuaciones. El Técnico coordinador convocó a los diferentes técnicos de las diferentes concejalías. Se quería que el técnico coordinador fuese el dinamizador del grupo para hacer las propuestas. Pero la realidad no es tan buena, porque la participación ha ido oscilando. Lo que se detectó es que se piensa que se trata de un proyecto que se realiza desde la Oficina y no entre todos y en ese caso es difícil que la gente asuma su parte de responsabilidad. El ayuntamiento se encuentra años luz de trabajar transversalmente. ${ }^{19}$

Elaboración de un Plan de igualdad de Oportunidades como instrumento para la implementación de la gestión transversal de género. Estrategia realizada desde el área de servicios sociales, responsable de las políticas de igualdad de oportunidades en género.

Desde 1993 existe un Plan para la igualdad, es uno de los Planes pioneros en Catalunya. Desde el equipo de gobierno se creía en la igualdad de género. Es

${ }^{18}$ Ayuntamiento catalán de izquierdas de una ciudad mediana.

${ }^{19}$ Ayuntamiento catalán de izquierdas de una ciudad mediana. 
un Plan transversal, no es una concejalía, se tiene que coordinar con todas las concejalías y servicios. Es desde este Plan que se hace todo el trabajo, es una apuesta importante por la transversalidad. ${ }^{20}$

La principal opción realizada por los ayuntamientos estudiados de la Lombardía ha sido crear una concejalía específica como 'assessorato per le pari opportunità'. La concejalía es la responsable directa de todo aquello que tenga relación con las políticas de igualdad en género. El modo de actuar dependerá del criterio y habilidad de la persona responsable de la misma, como es posible comprobar en las palabras de las responsables entrevistadas:

La concejalía de igualdad de oportunidades se ha creado hace 4 años, en el 2001, el año después de que el alcalde fuera elegido por primera vez. Y yo fui nombrada, soy la prima concejala de igualdad de oportunidades, no había nada, no existían políticas específicas sobre la igualdad de oportunidades, no se habían hecho proyectos pensados con una óptica de género ${ }^{21}$.

Es la primera vez que se ha instituido la concejalía de igualdad de oportunidades, en el sentido que no había existido nunca una concejalía de este estilo. El objetivo de esta concejalía es obviamente valorizar la subjetividad de las mujeres dentro de la ciudad, pero no solamente sobre la igualdad de oportunidades, sino también entendidas como la valorización de todos y todas, para asegurar la presencia de una paridad en su dignidad. ${ }^{22}$

Esta opción, la creación de una concejalía específica, también ha sido seguida por dos ayuntamientos catalanes. Ambos tienen una concejalía de la mujer; una de ellas pensada para trabajar de forma transversal, como explica su responsable: "La Concejalía de la mujer se ha creado y es transversal, realiza actuaciones directas al mismo tiempo que vigila la sensibilidad de las otras concejalías". ${ }^{23}$

\footnotetext{
${ }^{20}$ Ayuntamiento catalán de izquierdas de una ciudad pequeña.

${ }^{21}$ Ayuntamiento lombardo de derechas de una ciudad mediana.

${ }^{22}$ Ayuntamiento lombardo de izquierdas de una ciudad grande.

${ }^{23}$ Ayuntamiento catalán de izquierdas de una ciudad pequeña.
} 
Políticas municipales de género en Catalunya y la Lombardía...

La otra concejalía resalta la importancia de su trayectoria que demuestra una clara evolución para adaptarse a las nuevas necesidades de las mujeres y a la nueva visión de políticas de género:

La concejalía de la mujer se creó el 1995. Durante ese primer mandato se realizaron políticas asistenciales (...) El 1999 se consideró conveniente mantener la parte asistencial (...) y empezar a realizar actividades para sacar la imagen de mujer como víctima y usuaria de servicios sociales (...) Se buscaba mostrar un papel más activo de la mujer. ${ }^{24}$

El organigrama presente en los dos ayuntamientos lombardos que no tienen una concejalía de políticas para la igualdad de oportunidades han seguido los siguientes caminos. En uno de ellos el alcalde ha dado el encargo de actuar a favor de estas políticas y supervisar la actuación de las otras concejalías a la concejalía de cultura, responsable de "la carta de las mujeres" (la carta delle donne). No existe un asesorato específico porque se ha decidido así, su objetivo es lograr que todas las concejalías presten atención al tema, opción que se considera más fácil de conseguir si no existe un responsable natural de las mismas. La asesora de cultura nos los cuenta con estas palabras:

No existe una delega para crear una concejalía, y es por una razón precisa, porque la atención de género, es decir, la atención a la igualdad de oportunidades es transversal en todas las directrices dadas por el alcalde. Esto significa que si yo debo proyectar un barrio, por lo tanto también el concejal de urbanismo debe tener presente los tiempos de la ciudad relacionados con las mujeres, tener en cuenta los servicios que el barrio tiene que tener en correspondencia a la política de las mujeres. No se trata, consecuentemente, de limitarse a dar a un concejal la atención a las políticas al femenino. ${ }^{25}$

La concejalía responsable de estas políticas en el otro ayuntamiento es la de servicios sociales, que ha creado un 'Sportello Donna' (Servicio de Atención a las

\footnotetext{
${ }^{24}$ Ayuntamiento catalán de izquierdas de una ciudad mediana.

${ }^{25}$ Ayuntamiento lombardo de izquierdas de una ciudad grande.
} 
Mujeres) llevado por personal técnico. La persona responsable tiene toda la confianza del concejal, que deja en sus manos casi todas las decisiones sobre los temas de igualdad de oportunidades, como nos cuenta ella misma:

El punto más importante de este 'sportello' que nace el 1999 en la oficina de relaciones con el público fue que era capaz de escuchar las necesidades del territorio, siempre en relación a sus partes débiles y meter en red, es decir, dirigir o resolver el problema en una amplia red de sujetos, sean estos públicos o privados que trabajan en el territorio sobre estos aspectos. (...) Lo primero que se tiene que hacer cuando existe necesidad o diferencia es saber escuchar esta necesidad, esto ya tiene un primer impacto, sobretodo en los sujetos más débiles, sobretodo en las mujeres, los menores y los discapacitados. ${ }^{26}$

Una vez conocidas las opciones orgánicas existentes y con el objetivo de valorar la importancia que los ayuntamientos estudiados dan a las políticas de igualdad de oportunidades en género se presentará qué prioridad se ha dado a la materia, si existe personal técnico especializado y su relación con el movimiento de mujeres.

El nivel de prioridad se ha catalogado como bajo (cuando se trata de un tema que a pesar de existir preocupa muy poco al equipo de gobierno); medio (cuando dentro de los pocos recursos existentes en el mundo local se está intentando trabajar a favor de la gestión transversal de género) y alto (cuando se define el gender mainstreaming como una de las líneas programáticas de la política municipal).

\begin{tabular}{|c|c|c|}
\hline Prioridad & CATALUNYA & LOMBARDÍA \\
\hline BAJA & 1 & 2 \\
\hline MEDIA & 2 & 2 \\
\hline ALTA & 3 & 2 \\
\hline
\end{tabular}

\footnotetext{
${ }^{26}$ Ayuntamiento lombardo de derechas de una ciudad pequeña.
}

Cuestiones de género, 2008, nº 3, pp. 367/402 
Políticas municipales de género en Catalunya y la Lombardía...

PRIORIDAD BAJA. El ayuntamiento catalán que atorga poca prioridad al tema lo desarrolla desde la concejalía de servicios sociales mediante su Plan de Igualdad de oportunidades. Situación que casi se reproduce en el consistorio lombardo que trabaja estas políticas desde su "Sportello Donna" dentro de servicios sociales. El otro caso de la Lombardía tiene una concejalía específica, responsable de este tema, sin que exista la implicación general de todo el ayuntamiento.

PRIORIDAD MEDIA. Los ayuntamientos que dan una prioridad media a la materia son aquellos que intentan trabajar el tema de forma transversal siendo concientes que no se trata de una de las principales prioridades del ayuntamiento. En tres casos (1 catalán y 2 lombardos) se afronta desde una concejalía específica y en el cuarto mediante un técnico transversal.

PRIORIDAD ALTA. Finalmente, 4 de los 5 consistorios (los 3 catalanes y 1 lombardo) que afirman que se trata de una línea programática de sus políticas dan una elevada prioridad a la perspectiva de género. Han optado por no crear una concejalía específica y buscar otro mecanismo para implementar la gestión transversal de género. Mecanismos que ya han sido presentados.

Respecto a la existencia o inexistencia de plantilla para trabajar en estas políticas se observa la presencia de una persona, mínimo, en casi todos los ayuntamientos estudiados ${ }^{27}$. No es posible afirmar que en el $100 \%$ de los casos se trate de dedicación exclusiva a las políticas de igualdad de oportunidades en género, lo que hace dudar sobre su especialización en los temas de género.

Se han percibido importantes diferencias entre los ayuntamientos catalanes y los lombardos en su relación con el movimiento de mujeres. Los primeros no hacen

${ }^{27}$ En Catalunya solamente un ayuntamiento trabaja sin ninguna plantilla, siendo dos los casos en la Lombardía. 
ninguna referencia a los movimientos de mujeres, mientras que una parte de los segundos considera que es gracia a estos que se está trabajando el tema.

Las concejalas catalanas entrevistadas atribuye el diseño de políticas de igualdad de oportunidades en género a la voluntad política de su partido, sin realizar ningún comentario sobre el peso de la tradición feminista. Tres responsables de los ayuntamientos lombardos, en cambio, atribuyen la existencia de su concejalía y la realización de políticas de género a la lucha y el trabajo realizado por las mujeres de los partidos políticos:

Esta concejalía ha sido querida por esta administración en base a la experiencia habida en nuestra ciudad, una experiencia nacida el 1990, cuando se empezó con un núcleo de mujeres políticas, las mujeres electas crearon un organismo institucional llamado 'asamblea de mujeres electas' y empezaron a trabajar con los temas relacionados con la igualdad de oportunidades. ${ }^{28}$

Insistiendo por parte de una de las responsables que estas políticas son consecuencia de la historia de las mujeres de la izquierda:

Yo formo parte de una formación política de izquierdas de toda la vida, y las políticas de igualdad de oportunidades, antes se llamaban de paridad, forman parte de la historia de las mujeres, de la historia de las mujeres de la izquierda. Me siento orgullosa de ser una mujer de la izquierda italiana porque las políticas europeas, en parte, aquellas más ligadas a las políticas de conciliación $\mathrm{y}$ aquellas sobre los tiempos han nacido en Italia de un pensamiento político y filosófico de los años 70 de las mujeres de la izquierda italiana, que se han concretizado a mitad de los años 80 , en el 85 , cuando algunas de estas mujeres de la izquierda han ganado en los ayuntamientos y se han convertido en alcaldesas. ${ }^{29}$

Los otros tres ayuntamientos de la Lombardía (dos de ellos son gobernados por Forza Italia) comparten la posición catalana y consideran el diseño de estas políticas consecuencia de la voluntad política del partido.

\footnotetext{
${ }^{28}$ Ayuntamiento lombardo de izquierdas de una ciudad grande.

${ }^{29}$ Ayuntamiento lombardo de izquierdas de una ciudad mediana.

Cuestiones de género, 2008, nº 3, pp. 367/402
} 
Políticas municipales de género en Catalunya y la Lombardía...

\section{b) Plan de actuación}

En este aspecto se ha observado un comportamiento totalmente diverso por parte de los ayuntamientos catalanes y lombardos. Todos los ayuntamientos catalanes estudiados han diseñado un plan de actuación que guiará todas las políticas a realizar para incorporar la visión de género, hacer desaparecer las desigualdades aún existentes y favorecer una mayor paridad entre sexos. Opción no seguida por los consistorios lombardos, que no han elaborado planes de actuación, ni manifiestan la voluntad de hacerlo. Parece que se trate de un instrumento ajeno a la política italiana de igualdad de oportunidades entre mujeres y hombres, ya que tampoco encontramos ningún Plan a nivel nacional o regional lombardo.

Las concejalas catalanas han optado por crear un plan porque consideran que es un instrumento que facilita el cambio de mentalidad en la administración y asegura un mínimo de trabajo transversal. Los planes se diseñan para lograr los objetivos que el ayuntamiento se ha marcado en el ámbito de la igualdad de oportunidades entre mujeres y hombres. Algunas de las personas entrevistas lo exponen con mucha claridad:

La primera discusión que se tuvo sobre como se tenía que llamar, si se trataba de un plan de igualdad (no se aceptó porque existen diferencias que se tienen que respectar), plan de la mujer (tampoco porque no se trataba de políticas de mujeres) y se decidió nombrarlo Plan de equidad. En primer lugar se hizo un pequeño análisis de la realidad de la población por sexos y edades. En segundo lugar se elaboró un esquema de las líneas estratégicas para lograr los objetivos del plan: Igualdad hombre - mujer; Promover acciones para corregir discriminaciones; vigilar por la vida de las mujeres; y Promoción activa de las mujeres en la ciudad. Se trata de seis líneas estratégicas que recogen todo un sequito de acciones. (...) Algunas de las acciones previstas se repiten en distintas líneas estratégicas. Se han previsto indicadores para la evaluación, pero es un paso difícil, aún hay mucho trabajo por hacer. ${ }^{30}$

El proceso de creación del programa Plan de igualdad de oportunidades. Lo primero fue conocer la situación. (...) Estudio de la situación de la mujer en la

\footnotetext{
${ }^{30}$ Ayuntamiento catalán de izquierdas de una ciudad mediana.
} 
ciudad fue lo primero para detectar las demandas y poder planificar con todos los departamentos y periodificarlo. Se realizó un estudio cuantitativo (datos estadísticos) y uno cuantitativo mediante 10 grupos de trabajo, formados por 10 mujeres con 10 perfiles distintos (mujeres inmigrantes, mujeres del textil, mujeres empresarias,...). (...) Son tres los parámetros que guían la elaboración del Plan: máximo consenso político (aunque en el ayuntamiento tengamos mayoría absoluta); máxima cooperación interdepertamental; y concertación ciudadana (tanto en el proceso como en la toma de decisiones) con las entidades y el Consejo para la Igualdad de Oportunidades. Se redactó el documento base para enmarcar el camino. Se continuó buscando la discusión e la implicación. Finalmente se redactó el Plan de igualdad de oportunidades, que es un documento muy conceptual.(...) Se elaboró un Plano Operativo, que fue aprobado el 2002, con vigencia durante el 2003. El objetivo era ir más allá del plano conceptual. Es la apuesta para que las propuestas no se queden a nivel conceptual, para asegurar que realmente se actúe y no se pueda ir atrás a nivel de presupuesto o de decisión política. ${ }^{31}$

Los ayuntamientos de la Lombardía estudiados y con una coalición de izquierdas en el gobierno han elaborado o están diseñando su "Piano dei tempi e orari della città", su ley del tiempo, que será la base de importantes actuaciones con perspectiva de género. Este instrumento es totalmente ajeno a las instituciones catalanas, que ni siquiera comentan su necesidad o utilidad. Solamente un ayuntamiento catalán ha creado una "Red de Nuevos Usos de los Tiempos"32.

Es curioso ver que mientras se presenta como un tema importante en la mayoría de los consistorios lombardos estudiados, sea un punto inobservado por parte de las dos ciudades estudiadas que tienen una coalición de derechas en el gobierno.

Los Planes de los tiempos y horarios de la ciudad son un instrumento para adaptar los horarios de los servicios existentes a las necesidades de toda la ciudadanía, como nos cuenta una las responsables municipales:

La finalidad general de las políticas temporales es la de mejorar la calidad de vida de la ciudad. Armonizando los horarios de los servicios se podrá mejorar

\footnotetext{
${ }^{31}$ Ayuntamiento catalán de izquierdas de una ciudad grande.

${ }^{32}$ Ayuntamiento catalán de izquierdas de una ciudad mediana.

Cuestiones de género, 2008, nº 3, pp. 367/402
} 
Políticas municipales de género en Catalunya y la Lombardía...

su calidad y la calidad del tempo de los ciudadanos y las ciudadanas: tiempo obligado y tiempo libre, tempo dedicado al trabajo, al cuidado de la persona o de la familia. (...) Elaborar un Plan regulador de los tiempos y horarios de la ciudad entendido como un conjunto de orientaciones y acciones de carácter intersectorial que entra a formar parte de los otros programas como el urbanístico, el de movilidad, el de cultura, de comercio, de los servicios educativos, de las políticas sociales, del trabajo y del turismo ${ }^{33}$.

\section{c) Tipología de políticas}

Se ha realizado una división de las políticas para la igualdad de oportunidades y de género de los ayuntamientos estudiados en tres grandes grupos. El primer grupo está formado por las políticas dirigidas a las mujeres con el objetivo de luchar contra las desigualdades y discriminaciones por razón de género y de sexo aún existentes. Concretamente las administraciones municipales analizadas están realizando:

Acciones para prevenir y erradicar la violencia de género y para asistir a sus víctimas.

Creación de servicios de atención, información y asesoría para las mujeres.

$>$ Políticas que quieren incrementar la participación de las mujeres en la vida social y política de la ciudad, y que buscan revalorizar el papel desarrollado por las mujeres en la historia.

$>$ Políticas dirigidas a colectivos específicos de mujeres para luchar contra las desigualdades y discriminaciones que pueden ser objeto: mujeres empresarias, mujeres jóvenes, mujeres extranjeras, mujeres desocupadas mayores de 45 años, prostitutas.

El segundo grupo de políticas engloba las actuaciones realizadas que buscan introducir cambios que faciliten la realización de políticas transversales de género, es decir, para que sea posible tener en cuenta en el diseño de las políticas que los hombres y las

${ }^{33}$ Párrafo de las líneas Programáticas publicadas para el mandato 2004 - 2009 y aprobadas en el pleno del ayuntamiento (deliberazione consiliare del 16/02/2005). Ciudad gobernada por una coalición de izquierda con una población de entre 100.000 y 150.000 habitantes 
mujeres no siempre tienen las mismas necesidades y experiencias ${ }^{34}$. Se han observado las siguientes políticas pertenecientes a este segundo grupo:

> Políticas de sensibilización sobre diferencias y desigualdades de género dirigidas a la ciudadanía.

$>$ Políticas de formación en género, dentro y fuera de la administración.

Y finalmente, el tercer grupo está formado por las políticas transversales de género, aquellas políticas que ya se han diseñado teniendo en cuenta las necesidades y características de los hombres y las mujeres de la ciudad:

$>$ Planes de los tiempos y horarios de la ciudad.

$>$ Políticas municipales que ya han introducido la perspectiva de género en su diseño. A pesar que sea cierto que casi todos los ayuntamientos estudiados realizan políticas de los tres grupos, se observan diferentes corrientes de actuación entre los consistorios catalanes y lombardos, que pasamos a comentar.

En relación a las políticas dirigidas específicamente a las mujeres el tema de la lucha contra la violencia de género marca importantes diferencias entre ambos territorios. Todos los ayuntamientos catalanes estudiados presentan el tema de la violencia de género como una prioridad en sus agendas y han elaborado protocolos de actuación ${ }^{35}$ para erradicar este problema. Realidad no compartida en los consistorios lombardos analizados, donde las responsables entrevistadas no realizan ningún comentario relacionado con este tema, ausente en sus agendas institucionales. Solamente en dos casos se nos informa que el ayuntamiento ofrece apoyo a entidades sin ánimo de lucro de la ciudad que dan asistencia a mujeres víctimas de violencia familiar. Podremos apreciar esta diferencia en las palabras pronunciadas por dos de las personas entrevistadas. Mientras una de las concejalas catalanas expone:

\footnotetext{
${ }^{34}$ Sin olvidar que se trata de colectivos que no son homogéneos, dentro de los cuales existen grandes diferencias por razón de clase social, estructura familiar, nivel formativo, etc.

${ }^{35}$ Uno de los ayuntamientos catalanes analizados se caracteriza por estar trabajando el tema de la violencia de género, aunque no considera que se trate de un tema de políticas de igualdad de oportunidades en género, sino que lo incluye entre sus políticas de asistencia social.
}

Cuestiones de género, 2008, nº 3, pp. 367/402 
Políticas municipales de género en Catalunya y la Lombardía...

Se ha iniciado el Protocolo, en el que se han incorporado todas las concejalías, cada una se ha tenido que plantear una acción en esta campaña (que se ha realizado durante tres semanas y ha recibido el apoyo de la Diputación provincial). La realización del Protocolo sobre violencia domestica es un tema muy complicado, hay muchos actores (...) Es un trabajo lento porque depende mucho de las personas que forman esta comisión. ${ }^{36}$

Desde la Lombardía se comenta lo siguiente:

Concretamente existen dos asociaciones (...) con las que colaboramos de forma muy estrecha. Una es sobre la problemática de la violencia dentro de las familias, con tutela de mujeres y menores, es un centro de servicios dedicado a las mujeres maltratadas. Y esta es una asociación con la que los servicios municipales colaboran ${ }^{37}$.

Las otras diferencias que se han detectado dentro de este primer grupo de políticas son de menor importancia. Los ayuntamientos catalanes dedican una mayor cantidad de recursos que los lombardos a las políticas que intentan dar un papel más activo a las mujeres en la ciudad y que buscan revalorizar su papel en la historia de la misma. Mientras que los ayuntamientos Lombardos resaltan, en general, la importancia que tiene el ámbito de la formación y el trabajo; ámbito menos recurrente en los consistorios catalanes. En ambos territorios se ofrece información y asistencia a las mujeres, buscando la mejor manera para darles a conocer sus derechos. En un ayuntamiento de la Lombardía se ha escogido un modo curioso y bastante discutible de realizar esta tarea, creando una "Parity Card" con financiamiento europeo:

Hemos elaborado una tarjeta (...) "parity card" (...), es como una tarjeta de crédito, nominativa, que ha sido enviada a todas las mujeres de la ciudad (...) el objetivo de esta tarjeta es el de dar la oportunidad a las mujeres de acceder a los servicios con un precio particular, a veces gratuitamente, y no son solamente servicios de lavandería con descuento, de libros comprados con

\footnotetext{
${ }^{36}$ Ayuntamiento catalán de izquierdas de una ciudad mediana.

${ }^{37}$ Ayuntamiento lombardo de izquierdas de una ciudad grande.
} 
descuento, sino que también hay asistencia legal, un conjunto de servicios para las mujeres que a lo mejor no tienen la formación suficiente que les permita moverse tan bien como otras, que a lo mejor tienen una situación familiar que se quieren separar y no saben donde ir, que no tienen dinero para pagarse un abogado. Y después realizar convenios con médicos. (...) Se pensaba en aquellos ámbitos que ayudan a estar bien, a vivir bien, dando la posibilidad de ir a un negocio y que este tenga, por ejemplo, un espacio donde dejar a los niños. Por lo tanto hemos aparejado a esta tarjeta una serie de convenios con el objetivo de activarla, pero las cosas con más relevancia son aquellas más difíciles de lograr. ${ }^{38}$

Entrando en el segundo grupo, las políticas municipales dirigidas a facilitar la introducción de la gestión transversal de género en los ayuntamientos, se observa una cierta paridad en las actuaciones de ambas regiones. Existen gobiernos que optan por la sensibilización y otros que apuestan por la formación en los dos territorios.

Es interesante resaltar la presencia de tres ayuntamientos que afrontan estas políticas de modo diverso. Por un lado, en Catalunya existe un consistorio que centra toda su apuesta por las políticas de género en la formación interna de la administración, quedando los otros dos grupos de políticas a niveles testimoniales. En palabras de su responsable:

Trabajo transversal dentro del propio ayuntamiento. Se busca que sea un ejemplo para la ciudadanía. (...) Se ve la transversalidad en las políticas del ayuntamiento mediante la formación continua. ${ }^{39}$

Por otro lado, hay dos ayuntamientos de la Lombardía que no realizan ninguna política dirigida a facilitar el gender mainstreaming. Se trata de los dos únicos ayuntamientos estudiados que están gobernados por una coalición de derechas. Y esta ausencia no se debe a que se considere un proceso fácil de realizar como nos cuenta una de las responsables:

\footnotetext{
${ }^{38}$ Ayuntamiento lombardo de derechas de una ciudad mediana.

${ }^{39}$ Ayuntamiento catalán de izquierdas de una ciudad mediana.

Cuestiones de género, 2008, nº 3, pp. 367/402
} 
Políticas municipales de género en Catalunya y la Lombardía...

Veo que existe una cierta dificultad y existe porque a lo mejor se trata de una dificultad relacionada con la falta de conocimiento, relacionada con la falta de voluntad de conocer, a una infravaloración del fenómeno y al hecho que en este fenómeno se habla solamente en inglés, este 'mainstreaming' ha hecho enloquecer a medio mundo. ${ }^{40}$

Finalmente, en relación a las políticas de género, la gran diferencia entre los ayuntamientos catalanes y lombardos la encontramos en las políticas que pretenden reorganizar los tiempos y horarios de la ciudad para adaptarlos a la vida de sus mujeres y sus hombres. Ámbito de gran importancia en los ayuntamientos lombardos (excepto en los dos casos ya vistos) y casi ausente en las agendas municipales catalanas, donde solamente una persona comenta el tema sin darle una gran importancia.

Una de las asesoras lombardas entrevistadas nos demuestra mediante un ejemplo porque es posible afirmar que las leyes del tiempo pueden ser buenas políticas de género:

Le doy un ejemplo para que se entienda mejor. En el ámbito del plan del tiempo de nuestra ciudad uno de los momentos que he individuado es el momento del ayuntamiento como agencia que ofrece servicios a la ciudadanía. Nosotros no podemos tener un edificio que tiene algunas oficinas abiertas en la planta baja, otras en la primera planta y otras en la segunda, con gente que continuamente sube y baja, es un lío. Estamos trabajando en ello, dentro de uno o dos meses habremos terminado, habremos cerrado todas las oficinas de los pisos superiores $\mathrm{Y}$ habremos llevado todas las oficinas abiertas al público a la planta baja. (...) son las pequeñas atenciones, sin inventar grandes luchas de las mujeres, son atenciones de la ciudad que reconocen hombres y mujeres en grado de hacer cosas o de tener problemas. ${ }^{41}$

De todas formas se realizan políticas de género en ambas regiones, ya que la mayoría de los ayuntamientos estudiados (5 de 6 en Catalunya y 4 de 6 en la Lombardía) han diseñado políticas teniendo en cuenta que su ciudadanía está formada por hombres y mujeres con necesidades, a veces, no coincidentes. No existe ningún

\footnotetext{
${ }^{40}$ Ayuntamiento lombardo de derechas de una ciudad mediana.

${ }^{41}$ Ayuntamiento lombardo de izquierdas de una ciudad mediana.
} 
gobierno local que afirme que se trate de su modo habitual de trabajar, pero si que es una perspectiva que poco a poco se está introduciendo gracias, sobretodo, al compromiso general asumido y a la labor realizada por la persona responsable.

En Catalunya son 5 administraciones municipales las que afirman que la introducción de la gestión transversal de género se está realizando mediante el Plan para la igualdad de oportunidades entre hombres y mujeres. El Plan actúa como instrumento que obliga a tener en cuenta la pluralidad existente en la población de la ciudad para lograr objetivos específicos en las distintas áreas. Este rol se aprecia en las respuestas dadas por las personas entrevistadas a la pregunta de si se tiene en cuenta cuál es la realidad de los hombres y las mujeres de la ciudad antes y después de realizar toda política:

La presencia del Plan enmarca todas las políticas. Se ha incorporado en la filosofía y en la sensibilización del ayuntamiento. El Plan enmarca la realidad. Se aprobó por unanimidad y todas las actuaciones lo tienen en cuenta. ${ }^{42}$

Desde el ayuntamiento en cualquier proyecto grande que se realice se intenta introducir la visión de género, mirar el proyecte desde la perspectiva femenina. Por ejemplo, se hizo cuando se redefinió la trayectoria de los autobuses y cuando se discutió el Plan estratégico. No se hace siempre, pero se hace. Depende mucho de los temas. Por ejemplo, en temas de educación o economía es mucho mas fácil tenerlo en cuenta, porque los temas ya se prestan, en otros temas la dificultad es mayor. Se tiene en cuenta en proyectos grandes, eso siempre. En el resto vigila el Plan. El Plan vigila para que se tenga en cuenta el género. Por ejemplo, es el Plan quien vigila para que desde deportes se tengan en cuenta a las mujeres. (...) El gran salto cualitativo ha sido sobretodo en el hecho que los concejales y las concejalas han escuchado los planes y se han tenido que posicionar. ${ }^{43}$

En la Lombardía los ayuntamientos comentan que están introduciendo la perspectiva de género poco a poco y en aquellos ámbitos más predispuestos, ya sea por su temática o por la sensibilidad de su responsable. Se remarca la importancia de la

${ }_{43}^{42}$ Ayuntamiento catalán de izquierdas de una ciudad grande.

${ }^{43}$ Ayuntamiento catalán de izquierdas de una ciudad pequeña.

Cuestiones de género, 2008, nº 3, pp. 367/402 
Políticas municipales de género en Catalunya y la Lombardía...

labor realizada por la asesora, persona que asume un rol activo en este proceso de cambio, que será posible gracias a la presencia de un compromiso general de toda la administración. La elaboración de planes sobre los tiempos y horarios de la ciudad está facilitando este proceso. Es posible observar en las entrevistas realizadas que para cada ayuntamiento es más fácil trabajar en distintos ámbitos, según haya sido su trayectoria política e histórica y la sensibilidad de los y las políticos responsables:

Mi presencia en junta sirve para que haya, de alguna manera, siempre un control sobre las opciones administrativas. Las opciones administrativas muchas veces vienen hechas con un criterio que es el de la economicidad (...) porque la primera regla es no despilfarrar el dinero público, pero cuando yo escojo el criterio de la economicidad y le hago un ejemplo, elimino la línea del autobús que sale a la una de la noche y que acostumbran a usar solamente tres personas, yo he hecho una opción óptima según las leyes italianas, pero ¿que he hecho contemporáneamente? ¿a quién he penalizado? Seguramente no ha sido a un hombre adulto, autónomo y con coche, he penalizado a otras categorías de personas, de manera particular a las mujeres, a los adolescentes, a las personas que no tienen la posibilidad de moverse, entonces yo tengo que valorar esta opción (...) Yo querría llegar a un presupuesto de género (...) Es decir, de alguna manera me gustaría llegar a controlar el presupuesto del ayuntamiento de esta manera, evaluando cada vez. ${ }^{44}$

Significa que si tengo que proyectar un barrio, de manera que hasta el concejal de urbanismo tiene que tener en cuenta los tiempos de la ciudad en relación a las mujeres, tiene que tener presentes los servicios que el barrio tiene que tener en correspondencia a la política a favor de las mujeres. No se trata solamente de encargar a un concejal que se dedique a las políticas al femenino. (...) Tengo que decir que existe un departamento de estadística muy bueno, por lo tanto del punto de vista de conocer donde se encuentra la necesidad es muy fácil, porque existe un buen seguimiento de la evolución, de la dinámica de la población. (...) Es la primera vez que se ha pensado un argumento transversal con especial atención sobre los tiempos de la mujer, (...). Pero repito creo mucho en poder construir alguna cosa que no tenga por sí una connotación femenina, sino que acabe siendo realmente una práctica común. ${ }^{45}$

\footnotetext{
${ }^{44}$ Ayuntamiento lombardo de izquierdas de una ciudad mediana.

${ }^{45}$ Ayuntamiento de izquierdas de una ciudad grande.
} 
Para terminar con este apartado son tres los ayuntamientos que no están realizando políticas de género en el sentido expuesto. La administración catalana que se encuentra en este grupo se ha centrado en la formación de su personal político y técnico, considera que la realización de políticas que incluyan la perspectiva de género es un segundo paso que vendrá de forma bastante natural después del proceso formativo existente. Los ayuntamientos lombardos que no trabajan aún en esta perspectiva realizan políticas para la igualdad de oportunidades entre mujeres y hombres dirigidas a las mujeres para ayudarlas en un mundo diseñado a medida del sexo masculino. Han introducido el gender mainstreaming en su discurso político, pero no en su manera de hacer políticas.

\section{3 Cambio en los actores políticos}

Aunque sea posible observar una cierta voluntad administrativa favorable a la participación ciudadana en estas políticas, aún no se puede afirmar, en la mayoría de los casos estudiados, que la ciudadanía y más concretamente las mujeres de la ciudad, sean un actor activo e importante en estas políticas.

En Catalunya 2 ayuntamientos consideran la participación ciudadana como uno de los principales ejes de sus políticas para la igualdad de oportunidades en género, lo que no significa que se haya logrado. En estos dos casos se ha creado un Consejo Municipal de Mujeres compuesto por representantes de todas las asociaciones que, de alguna manera, trabajan para la igualdad de oportunidades entre mujeres y hombres. El órgano tiene carácter consultivo y su utilidad práctica depende de la voluntad política de la concejala responsable de las políticas, ya que sus propuestas o opiniones no son vinculantes:

Consejo municipal para la igualdad de oportunidades (periodicidad: cada dos meses). Va más allá de las asociaciones de mujeres, se buscaba romper con esta exclusividad. Su composición es plural, pueden formar parte de ella todas las entidades que trabajen para la igualdad de oportunidades. Un problema que 
Políticas municipales de género en Catalunya y la Lombardía...

se detecta cuando se habla de consejo es que son pocas las entidades que trabajan para la igualdad de oportunidades y que la presencia femenina en lugares de importancia es poca. ${ }^{46}$

Existe un tercer caso en que a pesar de haber creado un Consejo de Igualdad que trabaja de forma muy activa como foro de debate, no se trata de un actor clave en el diseño de las políticas por su particular forma de actuar:

Consejo municipal de igualdad. Se encuentran una vez por trimestre en base a sus estatutos, pero se reúnen siempre que consideran que hace falta. Es el foro donde se produce el debate con la oposición y la ciudadanía. Es un buen lugar para hablar de los temas que interesan. Durante los dos primeros años se habló mucho de mujeres maltratadas. (...) Se decidió que se escogerían cada año dos temas para tratar. Es necesario ser prácticos, la gente del Consejo tiene otras obligaciones y vale la pena ser concreto y efectivo para que tenga un contenido real. $^{47}$

En la Lombardía son tres los ayuntamientos que manifiestan que la participación ciudadana es importante y que han creado una comisión (Commissione per le Pari Opportunità). En uno de los casos se trata de una comisión consultiva municipal que se encarga de dar opiniones a consultas y está formado solamente por representantes de los partidos políticos presentes en el ayuntamiento. Las otras dos comisiones se caracterizan por plantear al plenario del ayuntamiento (consiglio) y al equipo de gobierno (giunta) propuestas y observaciones. Su composición, en ambos casos, está formada por las mujeres electas de la administración municipal y de representantes de las asociaciones que trabajan en el territorio. Esta Comisión se configura como un elemento central en el diseño de las políticas, al ser la responsable, en varios casos de realizar las propuestas o de trabajar los temas que interesan.

\footnotetext{
${ }^{46}$ Ayuntamiento catalán de izquierdas de una ciudad grande.

${ }^{47}$ Ayuntamiento catalán de izquierdas de una ciudad pequeña.
} 


\begin{abstract}
Nosotros tenemos el estatuto que prevé la constitución de la comisión para la igualdad de oportunidades (CPO), que ha sido instituida en base al estatuto por primera vez en el 1991 (...). Nuestra CPO según su reglamento está formada por todas las mujeres electas en el plenario del ayuntamiento y las mujeres concejalas, y de 10 mujeres de la sociedad civil, es decir, de 10 mujeres que presentan su currículum y después la junta escoge entre las candidatas presentadas. (...) a veces las propuestas que finalmente llevamos a cabo nos llegan, como sería el proyecto de la conciliación de los tiempos, proviene de la concejalía (...) Pero normalmente las propuestas llegan sobre la misma mesa, las analizamos, las escogemos, y provienen también de las mujeres de la comisión. ${ }^{48}$
\end{abstract}

Comparativamente las dos comisiones lombardas existentes tienen un rol más importante que los dos consejos catalanes en el diseño de las políticas municipales.

Para los otros tres ayuntamientos catalanes y lombardos se trata de un tema ajeno a su interés político.

\title{
V. Conclusiones
}

Del estudio realizado de los ayuntamientos catalanes y lombardos se puede apreciar la presencia, en ambos territorios, de cambios institucionales y organizativos para facilitar la implementación de la gestión transversal de género.

En primero lugar, se han detectado cambios en el proceso político, el discurso del gender mainstreaming se ha introducido en las agendas municipales, transformándose en un Plan para la Igualdad de Oportunidades entre mujeres y hombres aprobado en el plenario del ayuntamiento en Catalunya y en un encargo explícito del alcalde a la concejala de políticas de igualdad en la Lombardía. A pesar de estas diferencias en ambos casos se remarca que el éxito depende, sobretodo, del trabajo y la perseverancia de las concejalas y asesoras responsables de la introducción de la

\footnotetext{
${ }^{48}$ Ayuntamiento lombardo de izquierdas de una ciudad grande.
} 
Políticas municipales de género en Catalunya y la Lombardía...

perspectiva de género en el ayuntamiento. Siendo la sensibilidad personal un factor clave para que se elaboren, desde cualquier departamento, políticas de género.

En segundo lugar, se han introducido cambios en los mecanismos políticos existentes. Los ayuntamientos catalanes han apostado por la creación de órganos especialmente pensados para trabajar transversalmente, mientras que los consistorios lombardos han preferido seguir con la opción más tradicional, crear una concejalía específica responsable de las políticas para la igualdad en género. En Catalunya las políticas han sido estructuradas dentro de planes de actuación que especifican los departamentos responsables, el período de ejecución, presupuesto, criterios de evaluación, etc. Opción inexistente en la Lombardía. Ambos territorios están realizando políticas para erradicar las desigualdades de género y políticas que ya introducen la perspectiva de género en su diseño, pero prestando una especial atención a aspectos diversos. En Catalunya la lucha contra la violencia de género se establece como una prioridad municipal, preocupación ajena a las agendas municipales lombardas. Por su parte las ciudades lombardas trabajan muy seriamente sus planes sobre los tiempos y horarios de la ciudad; planes inexistentes en Catalunya.

Finalmente y en relación a los cambios producidos en los actores participantes en el diseño de las políticas de igualdad de oportunidades en género, es posible afirmar que es un aspecto que necesita mejorar notablemente en ambos territorios. A pesar de considerar la participación ciudadana importante no se han creado instrumentos válidos que sean operativos y que permitan afirmar que las mujeres tienen un papel activo en el diseño de las mismas o que se ha logrado una mayor participación femenina en política. 


\section{Bibliografía}

ARRANZ, F. (ed.) 2000. Las políticas públicas a favor de las mujeres. Madrid: Instituto de Investigaciones Feministas UCM.

BIMBI, F. 2000. "The Family Paradigm in the Italian Welfare State (1947-1996)" in M.J. González, et. al (eds.) Gender, Inequalities in Southern Europe. Women, Work and Welfare in the 1990s. London: Frank Cass Publishers.

BLANCO Y GOMÀ (ed.). 2002. Gobiernos locales y redes participativas. Barcelona: Ariel.

BORJA Y CASTELLS. 1997. Local y global. La gestión de las ciudades en la era de la información. Madrid: Taurus.

BORJA, J. 1988. Estado y ciudad: descentralización política y participación, Barcelona: PPU.

BRUGUÉ, Q. 2002. “Nuevos Ayuntamientos, concejales diferentes: del gobierno de las instituciones al gobierno de las redes”, Revista Española de Ciencia Política, 7: 9-38.

BUSTELO, M. 2004. Las políticas públicas de igualdad de género en España. Madrid: Catarata.

COMISIÓN DE LAS COMUNIDADES EUROPEAS -SEC(2006) 275- Comunicación de la Comisión al Consejo, al Parlamento Europeo, al Comité Económico y Social Europeo y al Comité de las Regiones. Plan de Trabajo para la igualdad entre mujeres y hombres. 2006-2010. Bruselas, 1.3.2006, COM (2006) 92 final.

DE LEÓN, M. 2002. "Equívocos de la solidaridad. Prácticas familiaristas en la construcción de la política social española”. Revista Internacional de Sociología (RIS), 31: $137-164$.

FARGION, V. 2001. "Italy: Moving from the Southern Model", a P. Alcock y G. Craig (eds.) International Social Policy. Welfare Regimes in the Developed World. Hampshire: Palgrave. 
Políticas municipales de género en Catalunya y la Lombardía...

FERRERA, M. 2005. Welfare State Reform in Southern Europe: Fighting Poverty and Social Exclusion in Italy, Spain, Portugal nad Greece. New York: Routledge.

GELAMBÍ, M. 2005. "Les polítiques de gènere en els ajuntaments catalans: en procés de construcció”. Working Paper de l'ICPS. Barcelona: Institut de Ciències Polítiques i Socials.

GOMÀ CARMONA, R. 1996. "La reestructuración de los sistemas de bienestar a Europa". Revista Internacional de Sociología. 15: 95-120.

GRUPO DE ESPECIALISTAS EN MAINSTREAMING (EG-S-MS). 1999. Mainstreaming de género. Marco conceptual, metodología y presentación de buenas practicas. Madrid: Ministerio de Trabajo y Asuntos Sociales. Instituto de la Mujer.

HANNAN, C. 2003. «Promover la igualdad de género: experiencias de la ONU en la implementación de la estrategia de mainstreaming de género». Jornadas Internacionales de la Unidad de Igualdad y Género. Instituto Andaluz de la Mujer. Carolyn Hannan forma parte de la División on the Advancement of Women ONU DAW -

INSTITUTO DE LA MUJER. 2005. Estudio comparativo de los planes de igualdad de oportunidades entre mujeres y hombres autonómicos y nacionales. Año 2004. Madrid: IM.

LOMBARDO, L. 2003. "La europeización de la política española de igualdad de género", Revista Española de Ciencia Política, 9: 65-82.

NALDINI, M. 2000. "Le politiche a sostegno delle responsabilità familiari nei casi storici italiano e spagnolo". Inchiesta, 128: 99-104.

POLLACK Y HAFNER-BURTON. 2000. "Mainstreaming Gender in the European Union”. Harvard Jean Monnet Working Paper Series 2/00, Cambridge.

REES, T. 1998. Mainstreaming Equality in the European Union. London: Routledge.

SARACENO, C. 1993. "Elementi per un'analisis delle trasformazioni di genere nella società contemporanea e delle loro conseguenze sociali”, Rassegna Italiana di Sociologia, XXXIV (1):19-51. 
SUBIRATS Y GOMÀ (Coords.). 2001. Govern i Polítiques Públiques a Catalunya (1980-2000). Barcelona: Edicions UB i UAB.

TRIFILETTI, R. 1999. "Southern European welfare regimes and the worsening position of women”. Journal of European Social Policy. 9: 49-64.

VALIENTE, C. 1999. «El feminismo de Estado en los ayuntamientos de la Comunidad Autónoma de Madrid: creación y actuaciones». GAPP. 13-14.

-2006. El feminismo de Estado en España: El Instituto de la Mujer (1983-2003). Valencia: Institut Universitari d'Estudis de la Dona. Universitat de València VARELLA MARTÍ, R. 2001. «Las políticas de igualdad e el ámbito del trabajo: una propuesta de construcción teórica desde el género» en: R. Radl Philipp (ed.). Cuestiones Actuales de Sociología del género. Madrid: CIS, Universidad Santiago de Compostela.Vilanova. 